\title{
Inovação e estratégia de diferenciação da indústria de revestimentos cerâmicos: um estudo de caso sobre a empresa Portobello
}

\author{
Innovation and differentiation strategy in the ceramic tile industry: a case study of the Portobello \\ Company
}

Recebido: 20/10/2021 | Revisado: 25/10/2021 | Aceito: 03/11/2021 | Publicado: 05/11/2021

Lucas José de Sena Nascimento ORCID: https://orcid.org/0000-0001-8002-582X Universidade Federal de Campina Grande, Brasil E-mail: lucasjosedsena@gmail.com

Isabel Lausanne Fontgalland ORCID: https://orcid.org/0000-0002-0087-2840 Universidade Federal de Campina Grande, Brasil E-mail: isabelfontgalland@gmail.com

\begin{abstract}
Resumo
A Portobello, localizada em Tijucas, Santa Catarina - Brasil, é a maior empresa de revestimentos do Brasil. Até 1979, a sua produção tinha como foco a fabricação de álcool e açúcar, entretanto suas atividades foram diversificadas e a empresa passou a ter como propósito a fabricação e a venda de revestimentos cerâmicos. A empresa é caracterizada pela constante inovaçãoe realização de melhorias e adaptações em seus processos para atingir o mercado através de produtos sustentáveis cominovações e tecnologias de processo. O presente artigo é um estudo de caso que tem como objetivo apresentar como, diante da concorrência, a adoção de inovação e estratégias de diferenciação na Portobello contribuem para a obtenção de maior destaque e participação no mercado.
\end{abstract}

Palavras-chave: Portobello; Cerâmica; Inovação; Diferenciação.

\begin{abstract}
Portobello, located in Tijucas, Santa Catarina - Brazil, is the largest coating company in Brazil. Until 1979, its production was focused on the manufacture of alcohol and sugar, however its activities were diversified and the company started to have the purpose of manufacturing and selling ceramic tiles. The company is characterized by the constant innovation and realization of improvements and adaptations in its processes to reach the market through sustainable products with innovations and process technologies. This article is a case study that aims to present how, in the face of competition, the adoption of innovation and differentiation strategies in Portobello contribute to achieving greater prominence and participation in the market.
\end{abstract}

Keywords: Portobello; Ceramic; Innovation; Differentiation.

\section{Introdução}

De acordo com a Associação Nacional dos Fabricantes de Cerâmica para Revestimentos, Louças Sanitárias e Congêneres - Anfacer, o Brasil é um dos principais players do mercado mundial de revestimentos cerâmicos, ocupando a terceira posição em produção e a segunda posição mundial em consumo, além de ser o sétimo no ranking das exportações. Existem no país 60 empresas, 71 unidades fabris e 137 marcas, além disso, o setor é responsável por 6\% do PIB da indústria de material de construção. Desse modo, nos últimos anos, a indústria de revestimento cerâmico vem passando por transformações relacionadas a processos, produtos e eficiência. Com isso, a concorrência no setor aumenta diariamente e surge a necessidade de adotar diferentes estratégias para o crescimento e diferenciação das empresas do segmento. Nesse sentido, a Portobello é uma empresa que busca pela inovação e diferenciação para tornar-se mais competitiva frente ao mercado e seus concorrentes. 


\section{Metodologia}

Bocchi et al. (2004) caracterizam o estudo de caso pela investigação aprofundada de um objeto dada sua importância na totalidade em que está inserido e sua representatividade em um conjunto de fenômenos da mesma espécie. Dessa forma, o presente trabalho trata-se de um estudo de caso qualitativo, o qual consiste na avaliação da empresa Portobello, analisando os fatores que a influenciam, seus aspectos e as características de sua atuação, principalmente no que tange a inovação e as estratégias de diferenciação.

\section{Resultados e Discussão}

\subsection{A Portobello}

O grupo Portobello iniciou suas atividades em 1944 com o estabelecimento da Usina de Açúcar Tijucas - USATI, em São João Batista - SC, onde a produção era concentrada em álcool e açúcar. Entretanto, em 1979, no município de Tijucas SC, foi fundada por Cesar Bastos Gomes, a Cerâmica Portobello que surgiu da diversificação das atividades da USATI, e possui seus exercícios principais direcionados à industrialização e comercialização de revestimentos cerâmicos.

Atualmente é a maior empresa de revestimentos do Brasil, vende os seus produtos para o mercado interno e externo, por meio da Portobello Shop e do canal de vendas para engenharia. Outrossim, conta com equipes que trabalham com design e inovação, lançando tendências de arquitetura e decoração no Brasil e as encaminhando para o mundo, por meio do canal de exportação e da Portobello America. Pode-se apontar que a empresa possui cerca de 3.434 colaboradores, 30 milhões de metros quadrados produzidos anualmente e uma receita bruta de $\mathrm{R} \$ 1.4$ bilhões.

A Portobello apresenta como seu propósito "viver design, transformar ambientes e emocionar pessoas". Já a sua visão, é "ser líder brasileira e protagonista global em soluções de revestimentos para ambientes, através do design e inovação”. A seguir, apresenta-se a Figura 1 na qual se pode observar a fachada da empresa.

Figura 1 - Fábrica Portobello.

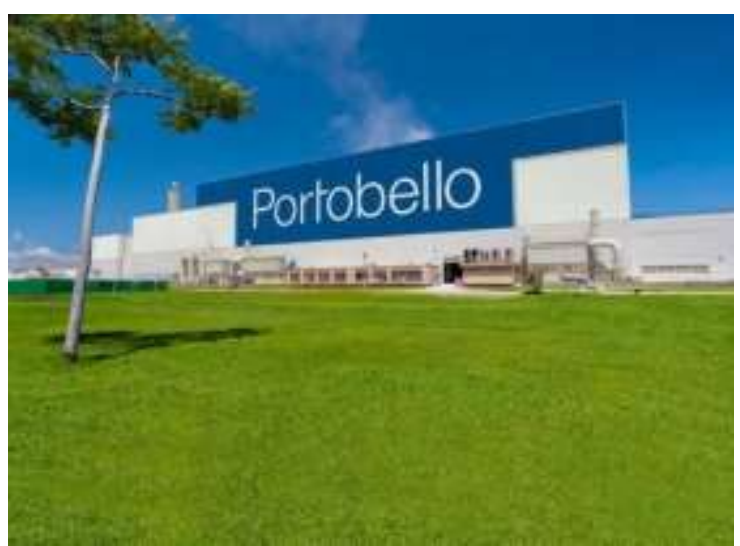

Fonte: Portobello (2021a).

\subsection{Produção}

A Portobello possui um processo de produção sustentável, que procura respeitar a biodiversidade e o uso dos recursos naturais. Assim, seu processo produtivo preocupa-se com quatro principais etapas: jazidas, água, energia e resíduos. Sua matéria prima é obtida por meio de fontes próprias e de terceiros. Ademais, trata da reestruturação das jazidas obedecendo a capacidade natural de cada área, seguindo as normas determinadas pela gestão de impactos sociais e de licenças das jazidas. 
Seus investimentos em tecnologias visam equipamentos que consomem uma menor quantidade de água. Dessa forma, a Portobello utiliza em seu processo produtivo um circuito fechado de água, onde toda a água utilizada na produção é tratada e reintroduzida ao processo. Dessarte, também ocorre a fabricação da cerâmica via seca, que não necessita de água no processo de moagem, sendo utilizada somente nos processos de esmaltação e polimento.

Acerca da energia, pode-se salientar que a fonte de energia da empresa é obtida pelo calor gerado no processo de produção das cerâmicas. Ainda, a empresa investe em energias limpas, as quais são $88 \%$ gás natural e $12 \%$ energia elétrica.

No tocante aos resíduos, por meio de uma reciclagem interna, a grande maioria são reincorporados ao processo produtivo, compondo a massa cerâmica. No entanto, o que não é possível aproveitar na massa é encaminhado para reciclagem externa, proporcionando uma economia circular e consciente.

Gráfico 1 - Produção Brasileira de Revestimentos Cerâmicos.

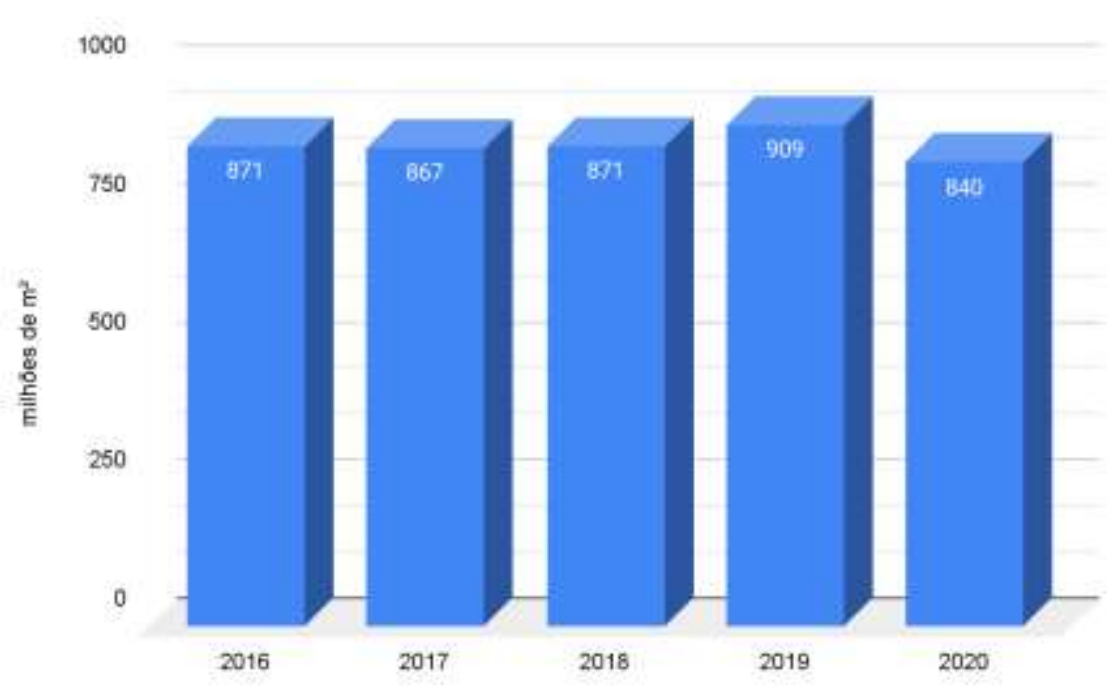

Fonte: Elaboração própria a partir dos dados da Anfacer (2021).

Conforme visto, no Gráfico 1, a produção de revestimentos incidiu ligeiramente, considerando-se a pandemia do COVID-19, contudo com boa margem devido sustentar-se compreensivelmente à construção civil. A pandemia, também, causou um franco impacto, no preço do metro quadrado de venda da casa própria, e a tomadas de ações foram de encontro à escorar preços estáveis até os novos sinalizadores de mercado atuarem (como reinvestimentos e mudanças tecnológicas de processos). De certa forma, houve uma reorientação dos investimentos para os pisos convencionais cerâmicos enquanto que o mercado de revestimentos e porcelanato estão ainda em aquecimento (Gráfico 2). 
Gráfico 2 - Tipos de Produtos Fabricados no Brasil.

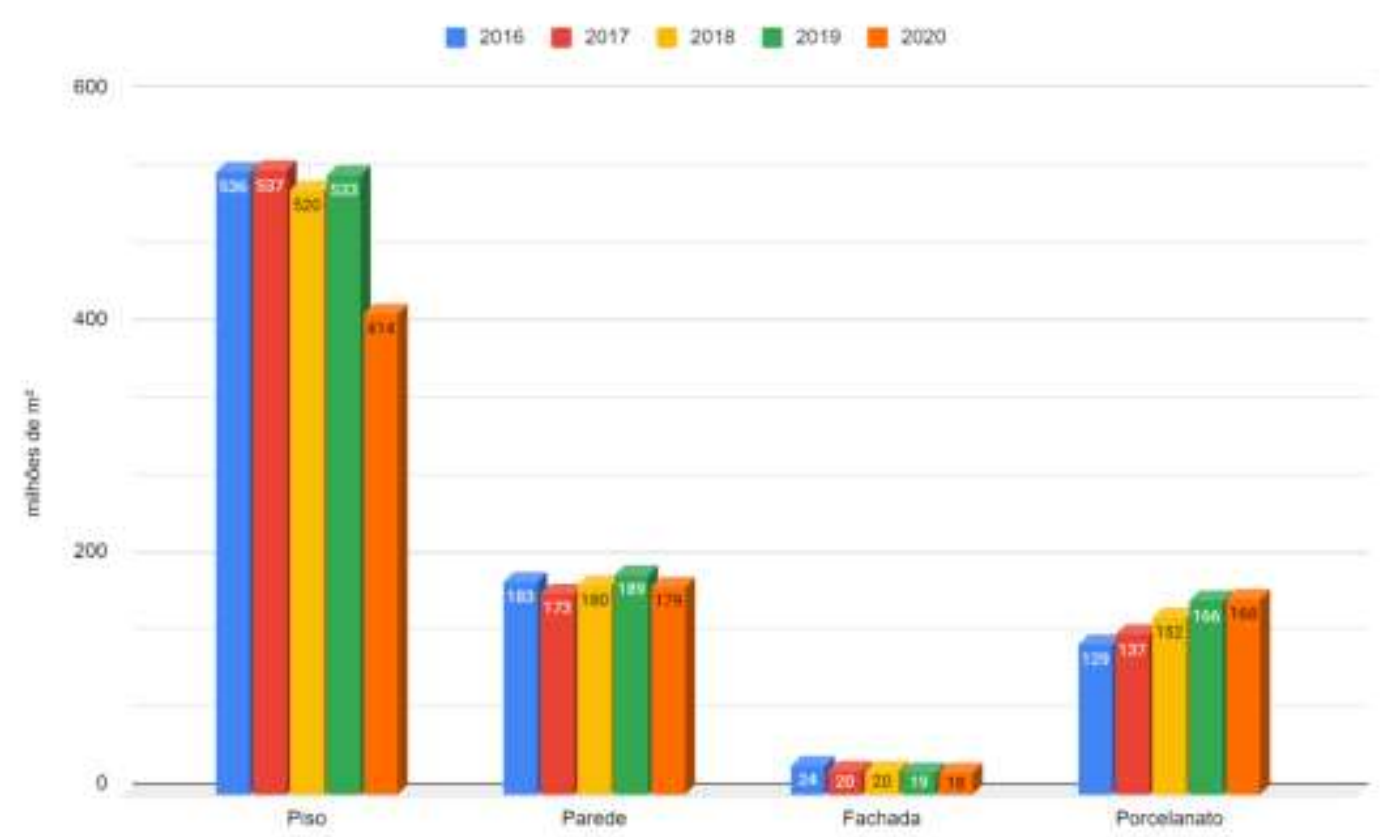

Fonte: Elaboração própria a partir dos dados da Anfacer (2021).

\subsection{Inovação}

De acordo com Soares (2007), quando a Portobello começou a produzir pisos do tipo porcelanato, muitos empenhos foram realizados para que a empresa atingisse seus objetivos. Para tanto, Glufke (2008) aponta que a empresa sempre esteve conectada à inovação tecnológica a fim de alcançar uma diferenciação entre as outras empresas nacionais, e também obter competitividade internacional.

Dessa forma, pode-se apontar que a Portobello adotou a inovação aberta, com um processo criativo que envolve a observação de novos comportamentos, pesquisas autorias, compartilhamento de habilidades e experiências, para conquistar os princípios de sustentabilidade e colaboração centrados na empresa. Além disso, Correia (2004), destaca que existem na empresa departamentos que têm a criação e a inovação como elemento fundamental.

Dessa maneira, a empresa inova em seu mix de produtos e também em sua gestão, assim, está sempre buscando transformar e desenvolver seus negócios por meio de um planejamento estratégico que visa simplificar e evoluir suas operações, por meio da redução do controle manual dos processos. (Battistotti et al., 2013).

A rede de franquias Portobello Shop, um dos exemplos de inovação efetuada pela empresa, consiste em uma rede de varejo especializado que oferece uma experiência de compra centrada no cliente, oferecendo revestimentos e complementos. Ainda, nas lojas Portobello Shop, o Sistema Lastras, permite que os profissionais do segmento, juntamente com seus clientes, tenham uma experiência de design com a marca, o que os tornam participantes no processo de determinação e compra. Além disso, existe a plataforma Archtrends, que por meio de referências e tendências, contribui com conteúdos técnicos e didáticos.

Diante do exposto, Battistotti et al. (2013) apontam que existem três fases de inovação: simplificação, estruturação e diferenciação. A primeira fase diz respeito à ampliação da eficiência operacional, que torna os processos mais rápidos e seguros; a segunda fase se refere à programação e implementação de melhorias nos processos de planejamento, que corroboram em melhor confiabilidade no atendimento aos clientes; a última fase, permite uma melhor relação com o cliente e 
com os canais de venda, o que resulta em um aumento da rentabilidade e do nível do serviço realizado.

\subsection{Estratégia de diferenciação}

A estratégia de diferenciação é utilizada pelas empresas com a finalidade de alcançar-se maiores lucros e participação de mercado. Assim, é possível destacar que não se trata somente de diferenciar produtos, e sim, diz respeito a tudo aquilo que permite a empresa atingir uma diferenciação sobre os concorrentes, como mudanças de processos e projetos (Canova, 2015; Possas, 1993).

A diferenciação de produtos acontece através de novos lançamentos e novos tipos de produtos que a empresa oferta no mercado, e a diferenciação de processos está relacionada com a busca por ganhos de eficiência e melhorias nos processos (Canova, 2015).

Para Battistotti et al. (2013), a Portobello baseia-se em uma estratégia de diferenciação, onde direciona seus esforços para ser reconhecida como a mais confiável do mercado de revestimentos cerâmicos. Com isso, a empresa obtém maior credibilidade e proximidade com o cliente por meio da rapidez e da confiabilidade, entretanto, sem deixar de se preocupar coma gestão de produtos e na eficiência operacional.

Desse modo, Canova (2015) salienta que na Portobello, as estratégias de diferenciação mostraram-se bastantes presentes, ocasionando transformações e atualizações, tanto em seus produtos, como também na logística, administração e marketing. É possível destacar que a empresa possui investimentos expressivos destinados à propaganda e marketing, que representam fatores fundamentais de uma forte estratégia de diferenciação.

Além disso, a empresa está continuamente apresentando novos produtos ao mercado, com mudanças rápidas das linhas e coleções, o que a torna diferenciada e inovadora, fazendo com que se posicione à frente dos outros concorrentes. Em seus produtos, a empresa possui como referência diversos tipos de materiais, que são destinados a vários ambientes, sejam eles internos ou externos, e ainda conta com vários formatos e tamanhos.

Seguindo, sobre a estratégia de diferenciação em termos de processo, é possível mencionar que a Portobello instalou diferentes canais estratégicos de distribuição e escoamento da produção voltados ao mercado interno e externo, onde cada canal tem características específicas de portfólios de produtos, serviços e política comercial (Canova, 2015).

Ademais, visando atender um público consumidor específico, a Portobello construiu uma fábrica no próprio parque industrial de Tijucas, destinada à produção de porcelanatos com maiores formatos. E nos últimos anos, a empresa tem buscado adotar a diferenciação de custos, que objetiva reduzir os custos com o intuito de melhorar a eficiência e aumentar a competitividade da empresa (Canova, 2015). A seguir pode-se observar, na Figura 2, as variedades de produtos disponibilizadas pela Portobello. 
Figura 2 - Categorias de Produtos Ofertados pela Portobello.

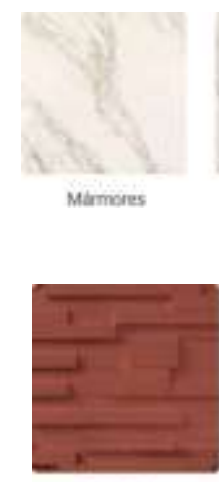

Tipolinhos

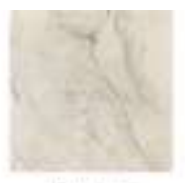

Quartanes

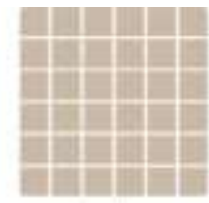

Pastikas

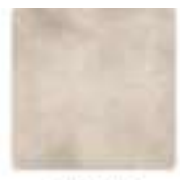

Cariomes

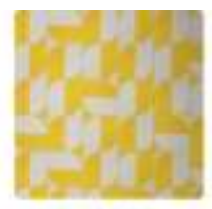

Azulejaris

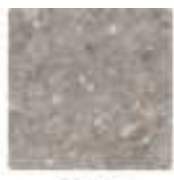

Prdtas

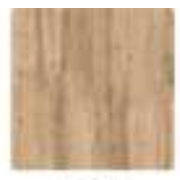

Naderas

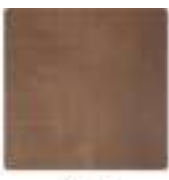

Metais

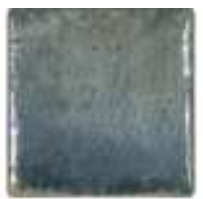

Decorados

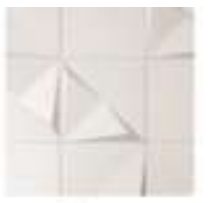

Reienos

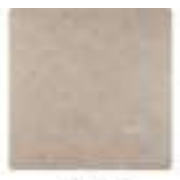

Terrazzo

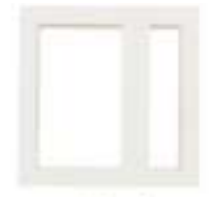

Cobogos

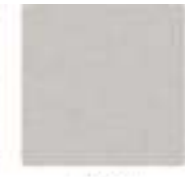

Snteticus

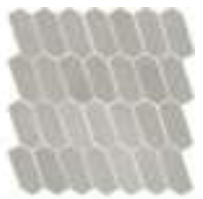

Mosaicos e Cortes Especiais

Fonte: Portobello (2021c).

\section{Considerações Finais}

Portanto, pode-se concluir que as inovações e as estratégias de diferenciação adotadas nos últimos anos pela Portobello possibilitam que a empresa se diferencie dos demais concorrentes, aumentando sua participação e crescimento no mercado. Assim, tais estratégias fortalecem a marca e permitem que a empresa apresente um acentuado crescimento frente ao mercado e os demais competidores, além de ter destaque em diferenciação, inovação, qualidade, criação de tendências e estilo.

Para trabalhos posteriores, recomenda-se o estudo de possíveis estratégias que a empresa possa adotar com a finalidade de dar continuidade em seu desenvolvimento e crescimento, de modo que vise respeitar os princípios de sustentabilidade e o uso consciente dos recursos naturais, além de levar em consideração as estratégias das empresas líderes no setor de revestimentos cerâmicos.

\section{Referências}

Alperstedt, G. D. et al. (2010) A internacionalização de empresas cerâmicas brasileiras: uma análise institucional. Revistas Perspectivas Contemporâneas. Edição Especial, 121-48.. https://revista2.grupointegrado.br/revista/index.php/perspectivascontemporaneas/article/view/906/311

Anfacer (2021a) Início. Website Anfacer. https://www.anfacer.org.br/

Anfacer (2021b) Números do Setor Cerâmico. Website Anfacer. https://www.anfacer.org.br/setor-ceramico/numeros-do-setor

Battistotti, H. R., Ardigó, C. M. \& Cardoso, O. R. (2013). Revestindo o caminho do serviço ao cliente por meio da logística: o caso da Portobello Shop. Revista Alcance. 20(4), 551-66. https://www.redalyc.org/articulo.oa?id=477748346009 Bocchi, J. I. et al. (2004) Monografia para economia. São Paulo: Saraiva.

Canova, G. (2015). Estratégias de crescimento empresarial: um estudo de caso da Portobello S/A. Trabalho de conclusão de curso de graduação, Universidade Federal de Santa Catarina, Florianópolis, SC, Brasil. https://repositorio.ufsc.br/bitstream/handle/123456789/15837 6/Monografia\%20do\%20Gustavo\%20Canova.pdf?sequence=1\&isAllowed=y

Correia, G. S. (2004). Avaliação do potencial da estrutura organizacional da cerâmica Portobello para o desenvolvimento da criatividade. Dissertação de mestrado, Universidade Federal de Santa Catarina, Brasil. https://repositorio.ufsc.br/bitstream/handle/123456789/87082/200011.pdf?sequence=1\&isAllowed=y

Glufke, R. M. (2008). A percepção do especificador de revestimentos cerâmicos sobre o papel do design gráfico na indústria: o caso Portobello Shop. Dissertação de mestrado, Universidade Federal de Santa Catarina, Florianópolis, SC, Brasil. https://repositorio.ufsc.br/bitstream/handle/ 123456789/91891/259530.pdf?sequence=1\&isAllowed=y

Gomes. J. (2015). Desempenho econômico-financeiro do segmento de revestimentos cerâmicos do sul catarinense: um estudo multicasos. Trabalho de conclusão de curso de graduação, Unesc, Criciúma, SC, Brasil. http://repositorio.unesc.net/bitstream/1/3643/1/Jucimar\%20Gomes.pdf

Lakatos, E. M; \& Marconi, M. A. (2003) Fundamentos de metodologia científica. (5a ed.). Atlas 312p.

Linares, R. (2004). Planejamento integrado das operações de venda e manufatura (S\&OP). O caso Portobello. Dissertação de mestrado, Universidade Federal de Santa Catarina, Florianópolis, SC, Brasil. https://repositorio.ufsc.br/bitstream/handle/123456789/86898/225063.pdf?sequence=1\&isAllowed=y 
Martins, P. R. (2019). Auditorias semanais dos produtos acabados da cerâmica Portobello (Relatório de Estágio/2019), Florianópolis, SC, Departamento de Química, Universidade Federal de Santa Catarina. https://repositorio.ufsc.br/bitstream/handle/123456789/202817/Tcc\%20Final\%20Pam ela\%20Rosa.pdf?sequence=1\&isAllowed=y

Meurer, A. P. (2008). Análise econômico-financeira de três empresas do setor cerâmico do estado de Santa Catarina. Trabalho de conclusão de curso de graduação, Universidade Federal de Santa Catarina, Florianópolis, SC, Brasil. https://repositorio.ufsc.br/bitstream/handle/123456789/126002/Cont abeis291525.pdf?sequence $=1 \&$ is Allowed $=y$

Petrazzini, I. M. B. (2014). Aumento de produtividade em uma linha de produção cerâmica. Monografia de especialização. Universidade Federal de Santa Catarina, Florianópolis, SC, Brasil. https://repositorio.ufsc.br/bitstream/handle/123456789/169969/PFC-20132-IgorPetrazzini.pdf?sequence=1\&isAllowed=y

Portobello (2021a) A Portobello. Website Portobello. https://www.portobello.com.br/institucional Portobello (2021b) A Portobello Shop. Website Portobello. https://www.portobello.com.br/portobello-shop Portobello (2021c) Início. Website Portobello. https://www.portobello.com.br/

Portobello (2021d) Inovação Aberta. Website Portobello. https://www.portobello.com.br/inovacao-aberta

Portobello (2021e) Sustentabilidade. Website Portobello. https://www.portobello.com.br/sustentabilidade

Possas, M. S. (1993). Concorrência e competitividade: notas sobre estratégia e dinâmica seletiva na economia capitalista. Tese de doutorado, Unicamp, Campinas, SP, Brasil. http://repositorio.unicamp.br/jspui/handle/REPOSIP/286024

Schmitt Neto, J. C. (2005). O processo de internacionalização de empresas sob uma ótica contextualista: um estudo de caso na cerâmica Portobello S/A. Dissertação de mestrado, Univali, Biguaçu, SC, Brasil. https://siaiap39.univali.br/repositorio/bitstream/repositorio/1637/1/Julio\%20Schmitt\%20Neto.pdf

Soares, T. T. (2007). A implantação da Cerâmica Portobello e as transformações estruturais e sócio-econômicas ocorridas no município de Tijucas. Trabalho de conclusão de curso de graduação, Universidade Federal de Santa Catarina, Florianópolis, SC, Brasil. https://repositorio.ufsc. br/bitstream/handle/123456789/122378/Economia293395.pdf?sequence=1\&isAllowed=y

Vargas, F. C., Kremer, A. W., \& Teixeira, B. (2019). Análise do comportamento assimétrico dos custos na empresa Portobello S.A. Anais Do Congresso Brasileiro De Custos - ABC. https://anaiscbc.emnuvens.com.br/anais/article/view/4655/4673 